\title{
Resisting emotional interference: Brain regions facilitating working memory performance during negative distraction
}

\author{
Alan Anticevic \\ Washington University, St. Louis, Missouri \\ Grega Repovs \\ University of Ljubljana, Ljubljana, Slovenia \\ AND \\ DEANNA M. BARCH \\ Washington University, St. Louis, Missouri
}

\begin{abstract}
Survival-relevant information has privileged access to our awareness even during active cognitive engagement. Previous work has demonstrated that during working memory (WM) negative emotional distraction disrupts activation in the lateral prefrontal regions while also engaging the amygdala. Here, using slow eventrelated fMRI, we replicate and extend previous work examining the effect of negative emotional distraction on WM: (1) We demonstrate that prefrontal regions showed activation differences between correct and incorrect trials during negative, but not neutral, distraction. Specifically, frontopolar prefrontal cortex showed more deactivation for incorrect trials faced with negative distraction, whereas ventrolateral prefrontal regions showed less activation; (2) individual differences in amygdala activity predicted WM performance during negative as well as neutral distraction, such that lower activity predicted better performance; and (3) amygdala showed negative correlations with prefrontal and parietal cortical regions during resting state. However, during negative distraction, amygdala signals were more negatively correlated with prefrontal cortical regions than was found for resting state and neutral distraction. These results provide further evidence for an inverse relationship between dorsal prefrontal cortical regions and the amygdala when processing aversive stimuli competes with ongoing cognitive operations, and further support the importance of the prefrontal cortex in resisting emotional interference. Supplemental materials associated with this article may be downloaded from http://cabn.psychonomic-journals .org/content/supplemental.
\end{abstract}

Emotional processing serves a highly adaptive function in the mammalian brain (Lang \& Davis, 2006; LeDoux, 2000; Öhman, 2005; Vuilleumier, 2005), allowing rapid deployment of attentional resources resulting in quick evaluation and decision making in the service of survival (Öhman, Flykt, \& Esteves, 2001). According to some theorists, emotional information may have privileged access to neural resources if attentional capacity is not fully depleted (Morris, Öhman, \& Dolan, 1998, 1999; Öhman et al., 2001; Pessoa, 2005; Pessoa, Japee, \& Ungerleider, 2005; Vuilleumier \& Pourtois, 2007), resulting in possible temporary disruption of cognitive goals (Dolcos \& McCarthy, 2006). However, at other times it may be more adaptive to sustain cognitive engagement regardless of incoming distraction (emotional or not), thus generating a more flexible behavioral repertoire-a function relying on frontoparietal cortical regions involved in "top-down" cognitive control (B. T. Miller \& D’Esposito, 2005; E. K. Miller \& Cohen, 2001).
One cognitive operation often used as a model of sustained cognitive engagement is working memory (WM), supported by a number of brain regions including dorsal frontoparietal cortical centers (Baddeley \& Hitch, 1994; Corbetta, Patel, \& Shulman, 2008; Corbetta \& Shulman, 2002; Curtis, Rao, \& D’Esposito, 2004; D’Esposito et al., 1998). Using a delayed WM task as a prototypical "cold" cognitive probe, Dolcos and McCarthy (2006) demonstrated a striking dichotomy in activation patterns between dorsal frontoparietal and ventral frontal-occipital areas when negative emotional distraction was presented. Dorsal frontoparietal regions (corresponding closely to the dorsal attention system; cf. Corbetta et al., 2008; Corbetta \& Shulman, 2002) showed a reduction in activity in response to negative, but not neutral, distractors. Dolcos and McCarthy suggested that these activation reductions may reflect dorsal regions temporarily being driven "offline" by regions responsible for detecting emotional salience 
such as the amygdala. In contrast, ventrolateral prefrontal cortex (VLPFC) showed an increase in activation to negative rather than neutral distractors. Importantly, a similar VLPFC region has been linked with effortful affect regulation (Ochsner, Bunge, Gross, \& Gabrieli, 2002; Ochsner \& Gross, 2005, 2008; Ochsner, Hughes, Robertson, Cooper, \& Gabrieli, 2009; Wager, Davidson, Hughes, Lindquist, \& Ochsner, 2008). Similarly, Dolcos and McCarthy suggested that elevated signals in the VLPFC region during WM might reflect an increased need for interference resolution arising from emotional distraction.

Apart from showing that negative distraction disrupts prefrontal cortical activity, it is critical to determine whether these regions are causally involved in resisting negative distraction. One way to provide evidence consistent with this hypothesis is to examine performancerelated activity during negative distraction. Dolcos and McCarthy (2006) showed lower dorsolateral prefrontal (DLPFC) activity for incorrect trials with negative distractors than for the average of all other trial types (e.g., correct negative distractors, correct and incorrect neutral distractors). Although this result is informative, it does not tell us whether activity in either dorsal or ventral prefrontal regions relates to accuracy selectively during negative distractors, or to accuracy under any condition (e.g., neutral distractors). Indeed, in a separate study, Dolcos, Kragel, Wang, and McCarthy (2006) showed that increased activity in VLPFC was associated with better performance during negative, but not neutral, distraction. However, they did not conduct these same comparisons for the dorsal frontal regions in the same study. Another way to characterize the role of PFC regions in resisting negative distraction is by examining the relationship between individual differences in PFC signals and individual differences in WM performance. Prior work found that individuals who showed less VLPFC activity reported higher levels of subjective distractibility (Dolcos \& McCarthy, 2006), but the association between individual differences in PFC activity during negative emotional interference and objective measures of WM performance remains unclear.

In addition to understanding the role of prefrontal regions in resisting negative interference, it is critical to understand the role of "bottom-up" regions, such as the amygdala, in contributing to negative distraction. Prior work demonstrated, using the same delayed WM task, that amygdala activation was highest following negative interference, which is consistent with the ever-growing body of evidence pointing to the amygdala as a critical node in detection of emotional salience, particularly information communicating possible threat (Dolcos, DiazGranados, Wang, \& McCarthy, 2008; Phan, Wager, Taylor, \& Liberzon, 2004; Phelps, 2006; Phelps \& LeDoux, 2005; Wager, Phan, Liberzon, \& Taylor, 2003; Zald, 2003). However, it is not yet clear whether the magnitude of amygdala activation to negative distraction is associated with performance in such situations. As with PFC regions, examining performance-related activity would further elucidate the amygdala's role in disrupting WM performance, specifically during negative interference.
Also, it is important to investigate whether individual differences in amygdala amplitude predict WM performance. Previous work has suggested that individual differences in amygdala activity correlate with self-reports of emotional distractibility (Dolcos \& McCarthy, 2006). However, it is not yet clear whether individual differences in amygdala signal are associated with objective performance measures during negative emotional distraction. Therefore, in addition to cortical foci, in the present study we examined the relationship between amygdala signals and WM performance.

In the above discussion, we considered cortical and subcortical regions separately. However, there is increasing awareness that putatively "cold" prefrontal (top-down) and "hot" emotional neural circuits may interact during emotional and cognitive processing (Pessoa, 2008; Wager et al., 2008). Recent advances in functional connectivity (fcMRI) analyses have allowed for more direct tests of the relationships between different neural regions during rest and task states (Mitchell et al., 2008). However, to our knowledge, there has been only one investigation examining amygdala trial-based connectivity patterns during negative distraction in the context of delayed WM function (Dolcos et al., 2006), focusing on the relationship between amygdala and bilateral VLPFC, which showed stronger correlations during negative than during neutral distraction. However, the relationship between the amygdala and other cortical regions (especially dorsal frontoparietal regions) during WM faced with negative distraction remains unclear. This question is of particular interest given the biased competition model of attention (Desimone \& Duncan, 1995), which would predict an ongoing competition for neural resources between the amygdala and the dorsal PFC, given their putatively different roles in the detection of sensory salience versus top-down task selection and control (for a review, see E. K. Miller \& Cohen, 2001; Phelps, 2006). One expression of such competition might also be direct inhibitory influence between the prefrontal cortex and the amygdala, which could be observed as negative coupling between these foci. Consistent with this hypothesis, a recent study by Mitchell et al. demonstrated significant negative correlation between the amygdala and the dorsal frontoparietal cortex during a shape identification task that contained both positive and negative distraction.

Furthermore, a recent investigation demonstrated negative correlations between the amygdala and what appeared to be the main components of the dorsal frontoparietal task network during resting state (Roy et al., 2009). Such resting-state findings raise interesting questions about whether negative amygdala-prefrontal coupling is equally present in both resting and task states. If, as discussed above, the negative correlations between the amygdala and prefrontal regions reflect a balance between responses based on emotional salience versus implementation of top-down goals (e.g., maintaining WM representations), it is possible that the amygdala dynamically adjusts its coupling with prefrontal nodes during task states requiring top-down control. Therefore, we specifically examined differences in fcMRI patterns between the amygdala and 
other brain regions during resting state, and during WM task faced with negative distraction.

To summarize, we examined signal patterns during active WM maintenance in dorsal and ventral lateral frontal regions, as well as the amygdala, while negative and neutral distractors were presented. The present study focused on the following goals: (1) Replicate previous findings showing different responses to negative versus neutral distraction in dorsal and ventral prefrontal regions as well as the amygdala; (2) examine whether prefrontal regions and the amygdala show either (or both) a trial-by-trial or an individual difference relationship with performance, specifically during negative distraction; (3) replicate restingstate amygdala-prefrontal fcMRI patterns and examine possible differences in connectivity patterns between these regions during WM faced with negative distraction.

\section{METHOD}

\section{Subjects}

Twenty-one neurologically intact right-handed healthy adults ( 8 male and 13 female; mean age, 24.95 years) were recruited from the Washington University Community by the psychology department subject coordinator and underwent neuroimaging data collection. All subjects completed and signed an informed consent approved by the Washington University IRB and were paid $\$ 25 / \mathrm{h}$ for their participation. An additional 21 neurologically intact righthanded healthy adults ( 13 male and 8 female; mean age, 22.52 years) completed resting-state fMRI data collection. We collected restingstate data from a different sample due to long duration of the experimental task (over $2.5 \mathrm{~h}$ in the scanner), which could have induced substantial subject fatigue and, in turn, excessive movement and loss of data quality during resting-state scans.

\section{Materials}

Subjects performed 180 trials of a version of the Sternberg WM delayed response task (Sternberg, 1969) with two levels of WM load (two or three complex geometric shapes) and three potential distractor types presented during the maintenance period of the WM task: (1) emotionally negative image; (2) visually complex neutral image; and (3) task-related geometric shape. We included the task-related geometric shape in order to further evaluate the specificity of the effects of negative distraction. Although neutral distractors help in this regard, they do not elicit the same level of performance impairment as do task-related distractions. Furthermore, prior work has shown that task-confusable distraction (i.e., distractors sharing task properties) was associated with increased signals in dorsal cortical regions rather than the decreased signals found for negative distraction (Dolcos et al., 2008). If negative distraction has a unique impact on PFC activity compared to other salient, but nonemotional interference, negative distractors should result in a different task-evoked signal pattern in both cortical and subcortical regions.

A portion of the trials did not contain a distractor (total of 30 blank trials randomized across the experiment) and were used to estimate distractor-free maintenance activity. The memory sets and task-related distractors were constructed from complex geometric shapes (Attneave \& Arnoult, 1956) that were difficult to verbally encode and were generated using a MATLAB algorithm (Collin \& McMullen, 2002). Memory set shapes and probes were set to pure black $(\mathrm{R}=255, \mathrm{G}=255, \mathrm{~B}=255)$, and task-related distractors were set to a shade of gray to be distinctive from the probes $(\mathrm{R}=$ $125, \mathrm{G}=125, \mathrm{~B}=125$ ). The negative and neutral visual distractors were selected from the IAPS stimulus set (Lang, Bradley, \& Cuthbert, 1999) and were equated on luminance, contrast, figure-ground relationships, spatial frequency, and color (Bradley, Hamby, Löw, \& Lang, 2007; Delplanque, N'diaye, Scherer, \& Grandjean, 2007;
Sabatinelli, Bradley, Fitzsimmons, \& Lang, 2005). All distractors were presented centrally, with a visual angle of $8.5^{\circ}$.

\section{Task Design}

The pool of 180 trials was divided into 90 high-WM-load and 90 low-WM-load trials. There were 25 task-related distractor trials, 25 negative distractor trials, 25 neutral, and 15 blank trials in each load condition. The trial sequence was pseudorandomized, with the constraint that no distractor type could appear in more than 3 consecutive trials (to avoid mood induction via negative distractors). The memory sets were presented centrally with a visual angle of $15.75^{\circ}$ for a duration of $4.4 \mathrm{sec}$, followed by an 8.8 -sec delay. The delay was followed by a 1.1 -sec presentation of the distractor (if present), then by a 6.6-sec postdistractor delay and a probe presented for $2.2 \mathrm{sec}$ (Figure 1). Each trial was followed by a 13.2sec fixation period to allow the hemodynamic response to return to baseline. Prior to the start of the experiment, each subject was presented with instructions explaining the task and given a brief (8-trial) practice session to demonstrate various trial combinations. The entire experiment was divided into 12 scanning sequences, each lasting $9.2 \mathrm{~min}$. During the scanning period, visual stimuli were presented through an LCD projector to a screen located behind the scanner, which the subject could see through an angled mirror located above the eyes.

fMRI scanning. All scanning occurred on a 3T Tim TRIO Scanner at Washington University Medical School. Functional images were acquired using an asymmetric spin-echo, echo-planar sequence, which was maximally sensitive to BOLD contrast $\left(\mathrm{T} 2^{*}\right)$ (repetition time $[\mathrm{TR}]=2,200 \mathrm{msec}$, echo time $[\mathrm{TE}]=27 \mathrm{msec}$, field of view $[\mathrm{FOV}]=256 \mathrm{~mm}$, flip $=90^{\circ}$, voxel size $\left.=4 \times 4 \times 4 \mathrm{~mm}\right)$. Each BOLD run contained 251 volumes consisting of 32 oblique axial images, which were acquired parallel to the anterior-posterior commissure. All structural images were acquired using a sagittal MP-RAGE 3D T1-weighted sequence $(\mathrm{TR}=2,400 \mathrm{msec}, \mathrm{TE}=3.16 \mathrm{msec}$, flip $=8^{\circ}$, voxel size $\left.=1 \times 1 \times 1 \mathrm{~mm}\right)$. Additionally, two restingstate BOLD runs (164 volumes, 35 slices per volume) were acquired for an independent sample of 21 subjects $(\mathrm{TR}=2,500 \mathrm{msec}, \mathrm{TE}=$ $27 \mathrm{msec}, \mathrm{FOV}=256 \mathrm{~mm}$, flip $=90^{\circ}$, voxel size $=5 \times 4 \times 4 \mathrm{~mm}$ ).

fMRI data preprocessing. The fMRI data preprocessing steps included: (1) compensation for slice-dependent time shifts; (2) removal of the first five images from each run during which the BOLD signal was allowed to reach steady state; (3) elimination of odd/even slice intensity differences due to interpolated acquisition; (4) realignment of data acquired in each subject within and across runs to compensate for rigid body motion (Ojemann et al., 1997); (5) intensity normalization to a whole brain mode value of 1,000 , but without bias or gain field correction; (6) registration of the 3-D structural volume (T1) to the atlas representative template based on 12 normal subjects represented in the Talairach coordinate system (Talairach \& Tournoux, 1988) using a 12-parameter affine transform and resampled to 1-mm cubic representation (Buckner et al., 2004; Ojemann et al., 1997); (7) coregistration of the 3-D fMRI volume to the structural image and transformation to atlas space using a single affine 12-parameter transform that included a resampling to a 3-mm cubic representation; (8) spatial smoothing using a 6-mm full-width at half maximum (FWHM) Gaussian filter.

General fMRI analysis. As a first step, a general linear model (GLM) approach was used to estimate task-related activity in each voxel for each subject without assuming a hemodynamic response shape (Ollinger, Corbetta, \& Shulman, 2001) and without differentiating correct and incorrect trials. The first 15 frames of each trial were modeled. Each of the eight conditions was modeled separately (two load levels and four distractor type trials), and the resulting beta estimates of event-related response at each trial time point (15 time points) were entered into a second-level analysis that treated subjects as a random factor. A second GLM model was computed that included accuracy as a covariate to enable examination of the within-subjects relationship between behavioral performance and brain activity. 


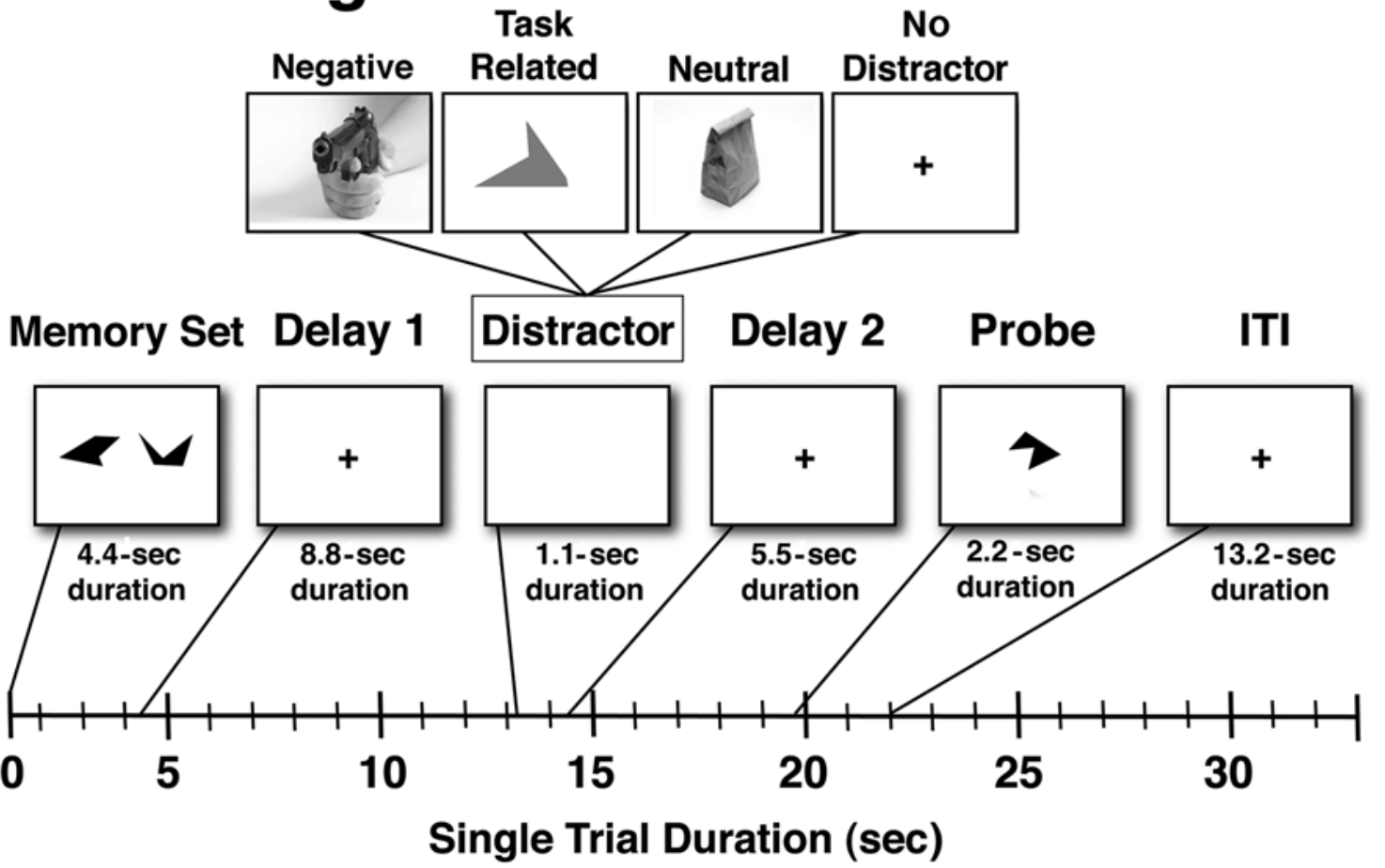

Figure 1. Task design. The overall layout of the task is shown along with different components and their onsets marked along the timeline. Each box represents a trial component with the duration marked below. First, subjects were presented with a set of complex geometric shapes, which they were instructed to memorize, followed by a delay. Next, during the middle phase of the trial subjects saw either (1) an emotionally negative distractor; (2) a task-related geometric shape distractor of a different color distinguishing it from the probe; (3) a neutral distractor; or (4) no distraction. This was followed by another delay. Finally, subjects were shown a probe and indicated via a button response whether it was part of the memorized set or not.

Given the focused questions concerned with effects of negative distraction on prefrontal activity, we identified cortical ROIs that showed either an increase or a decrease in activation during negative, rather than neutral, distraction. We used three analytic steps to isolate these regions. First, to isolate ROIs that met whole-brain false-positive correction criteria, we identified voxels showing significant differences in time courses across all conditions using a two-way repeated measures ANOVA with distractor type (four levels) and time (15 frames per trial) as factors, treating subjects as a random factor. This was done to identify voxels showing time course differences across different distractor conditions. Voxels that showed significant distractor type $\times$ time interaction and met a whole-brain $p<.05$ correction $(Z>3$ and a cluster size of at least 13 contiguous voxels) were considered for subsequent analyses. Second, to identify focal ROIs within the thresholded ANOVA map, we employed an automated peak-searching algorithm, delineating separate ROIs if they were more than $10 \mathrm{~mm}$ apart. These ROIs were limited to no more than $80 \mathrm{~mm}^{3}$, in order to preclude creating ROIs that spanned several functionally distinct cortical regions (Kerr, Gusnard, Snyder, \& Raichle, 2004; Michelon, Snyder, Buckner, McAvoy, \& Zacks, 2003). Third, to focus on ROIs showing modulation as a function of negative distraction, we computed a planned paired $t$ test on signals extracted from each of the identified ROIs for the two frames following the distractor presentation (average of Frames 8 and 9 in the trial most likely to reflect response to distractors) for negative versus neutral conditions. Only ROIs showing a significant difference in this $t$ test comparing negative with neutral distraction at $p<.01$ were considered in subsequent analyses (see Supplemental Table S1).
To isolate task-evoked amygdala signals, we applied an anatomical amygdala ROI mask based on the current sample, which was obtained using an automated subcortical segmentation process available through FreeSurfer (Fischl et al., 2002; Fischl et al., 2004). Specifically, we identified each individual's bilateral amygdala on the basis of anatomical images already registered to a common space. Next, we combined all the individual masks (inclusively, so that a voxel present in any individual subject's amygdala mask was present in the group mask) and downsampled the resolution to match the functional voxel size (i.e., $3 \times 3 \times 3 \mathrm{~mm}$; see Supplemental Figure S2). We then applied this bilateral amygdala mask to the ANOVA analyses described above to isolate thresholded voxels specifically within our anatomically defined amygdala regions. We also used the same anatomical amygdala mask to examine the relationship between amygdala signal and task performance.

fcMRI preprocessing. Prior to performing fcMRI analyses, all raw time series BOLD images were further preprocessed to remove possible sources of spurious correlations. All preprocessing, as well as further fcMRI analyses, was performed using in-house software implemented in MATLAB 7.4 and was based on previously published fcMRI techniques (Fox et al., 2005; He et al., 2007). (1) All images were spatially smoothed by $6-\mathrm{mm}$ FWHM Gaussian filter (as in the GLM computation above). (2) Images were temporally filtered using a high-pass filter with cutoff frequency of $0.009 \mathrm{~Hz}$ to remove low frequencies and scanner drift. (3) Modeled after the procedure employed by Fox et al., a set of nuisance regressors were removed from the signal using multiple regression: six rigid-body motion correction parameters, ventricle signal, deep white matter signal, and whole-brain signal. Whole-brain and ventricle regions 
were defined individually for each BOLD run on the basis of its first frame volume using an automated algorithm. Brain edge was identified using a fixed threshold. Ventricle centers were identified by peak intensities within a predefined search volume. Ventricle extent was identified by an iterative searching algorithm sensitive to large intensity changes using previously identified peaks as seeds. Eyes were excluded based on a predefined mask. As a final step, one layer of boundary voxels was excluded from both whole brain and ventricle regions to exclude any possible remaining overlap. All the nuisance regressors were also expressed as their first temporal derivative to remove their temporally shifted versions. All subsequent analyses were based on the residual signal after removal was carried out for the listed nuisance regressors.

Seed-based correlation map analysis. We wanted to examine the relationship between the amygdala and other cortical regions, during both resting state and the WM task. To examine the amygdala fcMRI during resting state, we computed a seed-based correlation map using 21 subjects from an independent sample that completed resting-state runs. Amygdala correlation maps were computed by extracting the average time series across all the voxels in each subject's individual anatomically defined bilateral amygdala ROI, which was then correlated with each voxel in the brain. We estimated group-level statistical significance by converting individual correlation maps to Fisher $Z$ maps and computing a voxelwise one-sample $t$ test (comparing the correlation against zero). To examine amygdala fcMRI in the context of the WM task, we computed the average BOLD signal value during the maintenance phase following distractor onset (average of Time Points 8 and 9) at each trial for each voxel in the image. These values were then concatenated into a 4-D (brain volume $\times$ trial) time series representing distractor response signal over all the trials. Using the same approach as in resting state, amygdala correlation maps were computed by extracting average values across all the voxels in the amygdala ROI and computing their correlation with each voxel in the brain. Importantly, the described approach (i.e., using isolated time points during each trial and not all the frames in a trial) effectively eliminates the influence of the task structure and prevents spurious correlations that would be induced by similarities in the overall task response across progression of the trial. In the analyses presented below, we focused on the average of Time Points 8 and 9, since they were most likely to reflect activity in response to distractors. As before, we estimated group-level statistical significance by converting individual correlation maps to Fisher $Z$ maps and computing a voxelwise one-sample $t$ test (comparing the correlation against zero). All statistical maps were appropriately corrected for multiple comparisons using cluster size Monte Carlo algorithms to ensure that the obtained foci met whole-brain false positive rates of $p<.05$. Lastly, all fcMRI analyses were based on the average of both correct and incorrect trials to maximize power given no a priori predictions with regard to connectivity differences as a function of performance.

\section{RESULTS}

\section{Behavioral Performance}

Using percent correct as the dependent measure (see Figure 2), we computed a two-way ANOVA (four levels of distractor factor and two levels of load factor), which showed a main effect of load $[F(1,20)=34.014, p<$ $.0001]$, no main effect of distractor type $[F(3,60)=1.864$, $p=.145$, n.s.], and a distractor type $\times$ load interaction $[F(3,60)=4.93, p<.005]$. Given previous work demonstrating that negative distractors confer WM performance costs, we computed planned $t$ tests with accuracy as the dependent measure comparing specifically neutral versus negative conditions under high and low loads. Negative distractors were associated with significant accuracy cost

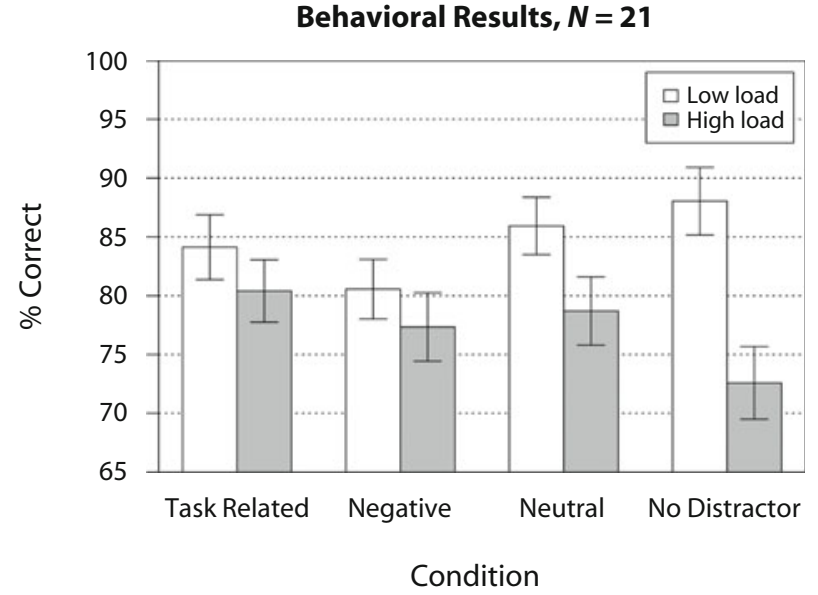

Figure 2. Behavioral results. Mean accuracy (expressed as percent correct) is shown for task-related, negative, neutral, and nodistractor conditions across two load levels. Error bars represent \pm 1 standard error of the mean.

in the low load $[t(20)=-3.48, p<.0007$, one-tailed $]$. There was no significant performance difference between negative and neutral distraction in the high load; however, consistent with prior work (Dolcos \& McCarthy, 2006), when collapsed across loads, $t$ test results indicated a significant WM cost for negative distraction, compared with neutral $[t(20)=-2.305, p<.017$, one-tailed]. Interestingly, the effects of emotional distraction were maximal at lower WM load levels. One possibility, supported by pilot data from our laboratory, is that the effect of negative emotional interference is not "detectable" in the context of a more difficult load manipulation. In other words, due to higher difficulty of the task and the need to maintain an accurate representation of more items in WM, all distraction may have been equally disruptive.

In addition, we computed the same two-way ANOVA with reaction time (RT), which indicated a main effect of load $[F(1,20)=7.87, p<.02]$, a main effect of distractor type $[F(3,60)=21.24, p<.0001]$, and no load $\times$ distractor type interaction $[F(3,60)=2.86, p=.12$, n.s.]. Lastly, as with the accuracy results, we computed planned $t$ tests using RT as the dependent measure, comparing specifically neutral and negative conditions under high and low loads. Consistent with accuracy results, negative distractors were associated with significant RT slowing in the low-load condition [ $t(20)=1.68, p=.05$, one-tailed]. However, as shown for accuracy, there were no significant RT differences between negative and neutral distraction in the high-load condition. Taken together, these results suggest that negative distractors were associated with significant accuracy and RT cost compared with neutral distractors and that these behavioral effects were most prominent under low WM load.

Surprisingly, performance in the distractor-free condition under high WM load was lower than in the distractor conditions. This pattern of behavioral results was unexpected and may reflect an artifact of the experimental design. One possibility is that since distractor trials were 
much more common than no-distractor trials, and presentation was randomized, subjects may have been surprised by the probe stimulus on the latter trials, especially under more difficult conditions (where WM traces may be more vulnerable). Additional out-of-scanner data collected with identical stimuli support this hypothesis; WM performance on distractor-free trials was considerably better when they were presented in a separate initial block than when they were mixed with all the distractor trials. Also, performance in blocked distractor-free conditions was better than in distractor trials at both WM load levels (see Supplemental Figure S7).

\section{Prefrontal Cortical Regions \\ Modulated by Negative Distraction}

Our first goal was to replicate previous findings and identify prefrontal cortical areas modulated by negative distractors. Our analyses (see the Method section for details) yielded 32 total foci with considerable similarities to previous findings (Dolcos \& McCarthy, 2006) (see Supplemental Table S1 and Supplemental Figures S1, S3, and S4). None of the identified ROIs showed a distractor type effect (e.g., negative vs. other types of distraction) that varied as a function of load. Thus, for ease of presentation, in subsequent analyses we averaged the activation across the two load levels.
As indicated at the beginning of this article, we focused specifically on frontal cortical foci showing activation modulation as a function of negative distraction reported by prior studies (Dolcos et al., 2008; Dolcos \& McCarthy, 2006). Right hemisphere foci are shown in Figure 3 and included DLPFC, frontopolar prefrontal cortex (aPFC), and VLPFC, closely matching those reported by Dolcos and McCarthy. Notably, left hemisphere effects were largely attenuated and are shown in Supplemental Figure S2, which is consistent with the visuospatial nature of the WM task (i.e., it may warrant more right hemisphere recruitment). In the absence of distraction, aPFC (Figure 3A, left panel) showed a lower signal pattern during the maintenance phase followed by a robust, transient response to the probe, also found by other groups investigating WM-related signals in this region (Leung, Gore, \& Goldman-Rakic, 2005). In contrast, DLPFC (Figure 3B, left panel) showed a marked response to the memoranda set during encoding, followed by a sustained, above-baseline signal during the maintenance phase and a prominent response to the probe, also consistent with prior work (Dolcos \& McCarthy, 2006). Both aPFC and DLPFC showed activation reduction during the delay period in response to negative when compared with neutral distractors [aPFC, $x=37, y=52, z=15, t(1,20)=-4.5, p<$ .00025 ; DLPFC, $x=40, y=34, z=33, t(1,20)=-5.53$, $p<.0001]$. Importantly, the reduced signal pattern in the

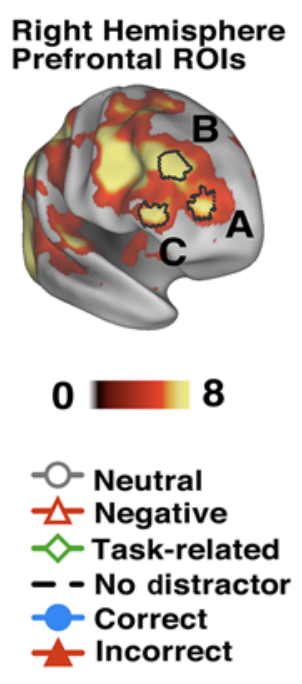

B
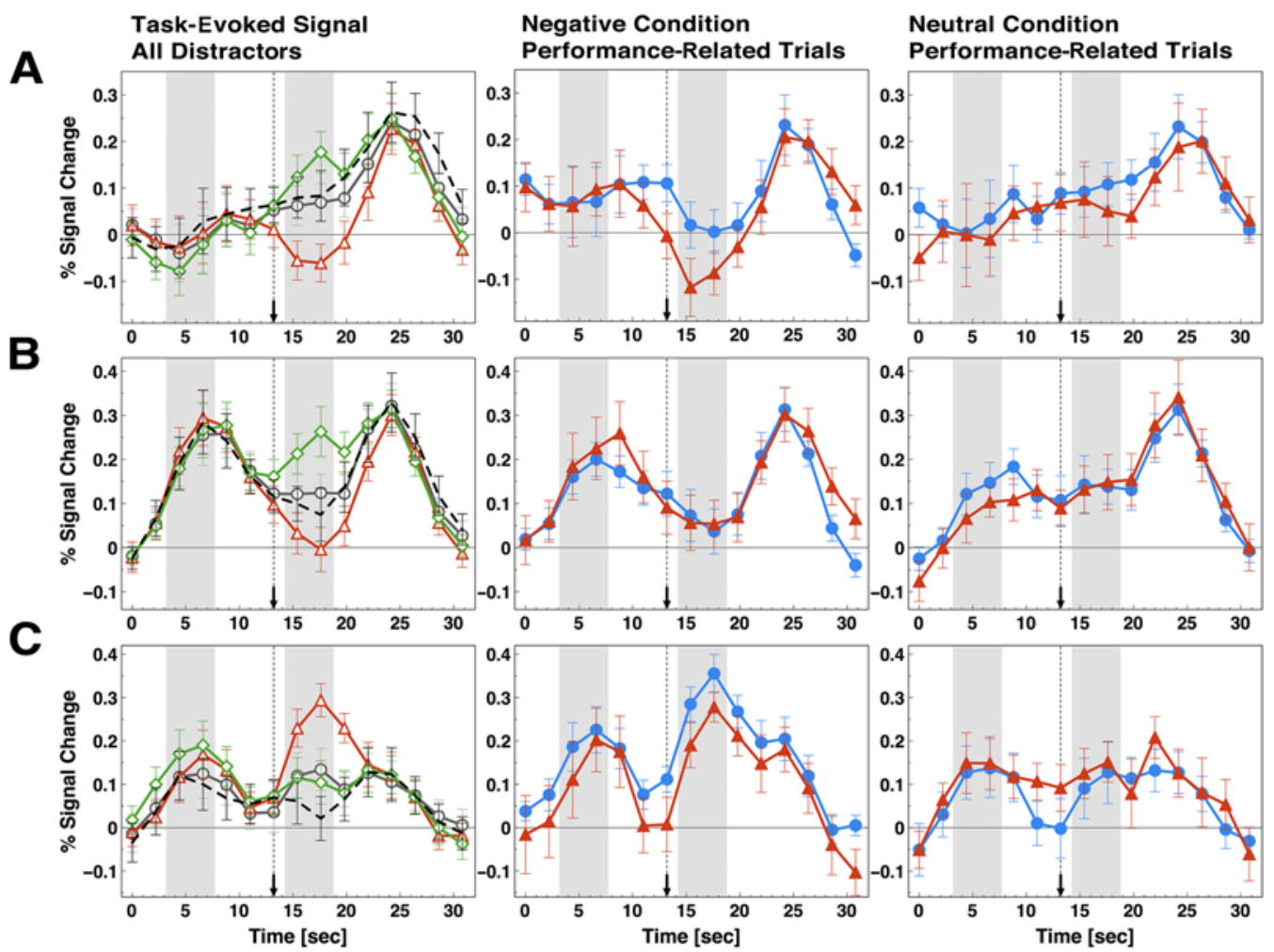

Figure 3. Task-evoked and performance-related time courses for right lateral prefrontal cortical foci. Event-related time courses are shown for right (A) anterior prefrontal cortex; (B) dorsolateral prefrontal cortex; and (C) ventrolateral prefrontal cortex. The far left set of graphs shows the task-evoked signals for neutral (circles), negative (triangles), task-related (diamonds), and no-distractor (dashed lines) conditions across all three ROIs. The middle panel shows performance-related time courses for the negative condition. The far right panel shows performance-related time courses for the neutral condition. Correct and incorrect time courses are shown with circles and triangles, respectively. Distractor onset is marked with a dotted vertical line ending in an arrow. 
dorsal PFC ROIs was specific for negative distraction, since other salient (task-related) but nonemotional distraction was associated with signal increases in dorsal PFC ROIs (see Figure 3). In contrast, VLPFC (Figure 3C, left panel) showed a signal increase in response to negative compared with neutral distractors [VLPFC: $x=51, y=33, z=14, t(1,20)=$ $2.83, p<.01]$. Again, this pattern was specific for negative distraction, given that task-related but nonemotional distractors were associated with minimal change in VLPFC signal. Taken together, these results replicate previously reported effects of negative distraction on PFC activity during WM (Dolcos et al., 2006; Dolcos \& McCarthy, 2006; Dolcos, Miller, Kragel, Jha, \& McCarthy, 2007). Next, we sought to extend these findings and test which of these regions show performance-related changes in activity, specifically during negative distraction.

\section{Relationship Between Prefrontal Activity and Performance}

We examined signal patterns for correct and incorrect trials for identified prefrontal cortical ROIs during negative and neutral distraction (Figure 3). The most prominent difference between correct and incorrect trials for the negative condition was observed in the aPFC ROI (Figure 3A), showing more deactivation for incorrect trials in response to negative, but not neutral, distraction. In addition, VLPFC showed higher signal for correct trials when faced with negative distraction. However, the nature of the signal as a function of performance in the VLPFC was different from aPFC. VLPFC (Figure 3C) showed less activation for incorrect trials during negative, but not neutral, distraction (whereas aPFC showed more deactivation). To confirm these findings statistically, we computed a paired $t$ test on the signal extracted from the prefrontal ROIs for the two frames following the distractor presentation (average of Frames 8 and 9 in the trial starting at time points of 15.4 and $17.6 \mathrm{sec}$, respectively), which indicated significantly lower signal for incorrect when compared with correct trials in the negative condition for both right aPFC ROI $[t(1,13)=$ $-2.51, p<.03$, two-tailed, Figure $3 \mathrm{~A}]$ and right VLPFC ROI $[t(1,13)=-1.94, p<.025$, one-tailed, Figure $3 \mathrm{C}]$. This same comparison failed to reach significance when examining correct and incorrect trials in the neutral condition in any of the above ROIs. However, the two-way interaction between emotion (negative vs. neutral distractor) and accuracy (correct vs. incorrect trials) did not reach significance for the aPFC $[F(1,13)=0.85, p=.37]$ and VLPFC $[F(1,13)=3.41, p=.087]$ ROIs. In addition, there were no differences between correct and incorrect trials in the DLPFC ROI for either negative or neutral conditions (Figure 3B, middle and far right panels). Also, aPFC, DLPFC, and VLPFC ROIs in the left hemisphere did not show significant differences between correct and incorrect trials (Supplemental Figures S3A-S3C). Overall, these results extend prior findings showing, in the same sample, that performance-related activation differences are especially evident in the frontopolar and ventrolateral prefrontal cortex when negative information interferes with performance.
In addition, we examined whether average activity in the same PFC ROIs was predictive of individual differences in WM performance across different conditions. We extracted the average BOLD signal across all voxels in each ROI (average of Frames 8 and 9) and computed a correlation across subjects with WM performance expressed as percent correct. Figure 4 shows the results for negative, neutral, and task-related distraction. Both aPFC and DLPFC showed an inverse relationship between average activation and WM performance, specifically for negative distraction but not for other conditions. To ensure statistical rigor, given the large number of computed relationships (12 total, including the amygdala), we employed a false discovery rate (FDR) correction (Benjamini $\&$ Hochberg, 1995). Both aPFC (Figure 5A) and DLPFC (Figure 5D) findings satisfied their respective FDR corrections $(q=0.05)$. However, although significant, the direction of this relationship was opposite to what we would have predicted, with less overall signal in aPFC and DLPFC being related to better performance. Importantly, these analyses averaged PFC activity for both correct and incorrect trials, but the results remained unchanged when correct trials only were examined (Supplemental Figures S6A-S6I).

\section{Is the Amygdala Modulated by Emotion During WM Maintenance?}

Figure 5A shows the bilateral amygdala ROIs identified using the time $\times$ distractor type interaction in the same manner as cortical foci (left, $x=-25, y=-8$, $z=-13,1,458 \mathrm{~mm}^{3}$ voxels; right, $x=25, y=-7, z=$ $-11,2,322 \mathrm{~mm}^{3}$; see the Method section for details). Figure $5 \mathrm{~B}$ shows the corresponding amygdala time courses. As in prior work, Figure 5B shows that highest amygdala signals were associated with negative, but much less so for other salient and distracting stimuli (e.g., task-related distraction). This pattern of result closely replicates the findings reported by Dolcos et al. (2008) in the context of a delayed WM task.

\section{Relationship Between the Amygdala and WM Performance}

As with cortical ROIs, we examined differences between correct and incorrect trials for the amygdala ROI. This analysis showed numerically higher signal for incorrect versus correct trials containing negative distractors, but the differences failed to reach significance. In addition to performing trial-by-trial analyses, we sought to examine whether individual differences in amygdala signal predicted WM performance. As shown in Figure 6, higher levels of bilateral amygdala signal were associated with worse WM performance across subjects for negative ( $r=$ $-.45, p<.05$, two-tailed) distractors (Figure 6A). However, this relationship was also present for neutral $(r=$ $-.63, p<.003$, two-tailed) and task-related $(r=-.57$, $p<.008$, two-tailed; Figures $6 \mathrm{~B}$ and $6 \mathrm{C}$, respectively) distractors. As for PFC ROIs, these analyses averaged amygdala activity for both correct and incorrect trials, but the results remained largely unchanged when correct trials only were examined (Supplemental Figures S6J-S6L). 


\section{aPFC}
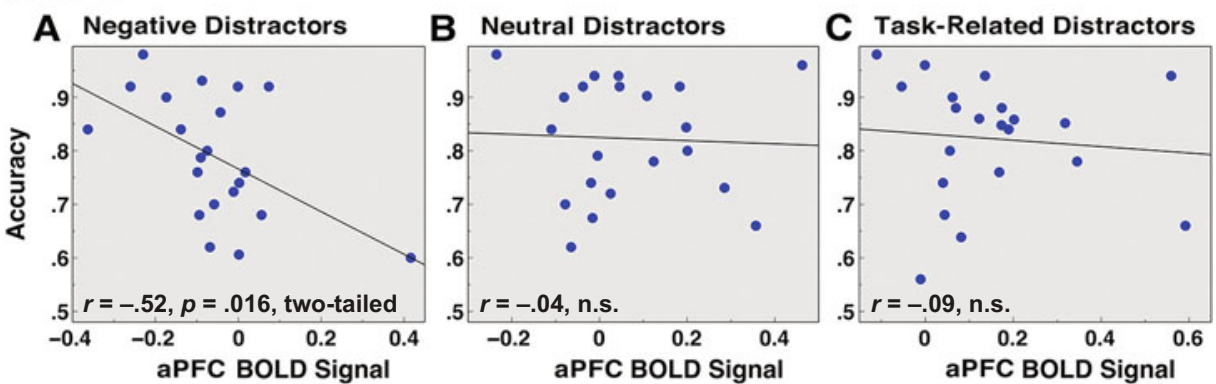

\section{DLPFC}

D Negative Distractors

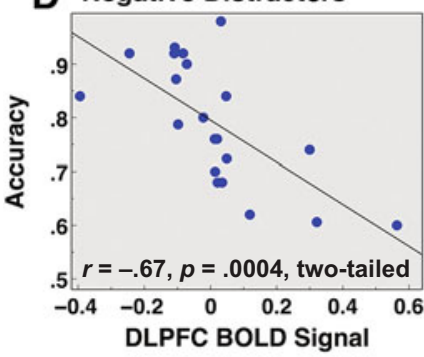

\section{VLPFC}

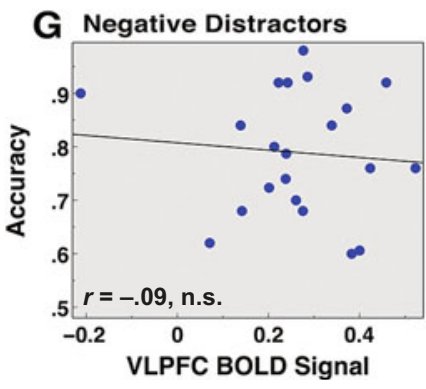

\section{E Neutral Distractors}

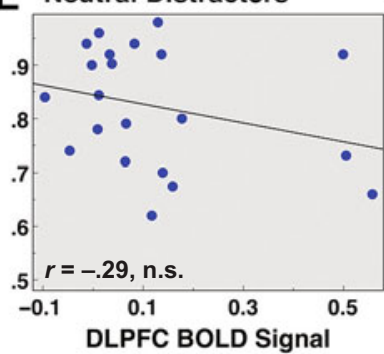

H Neutral Distractors

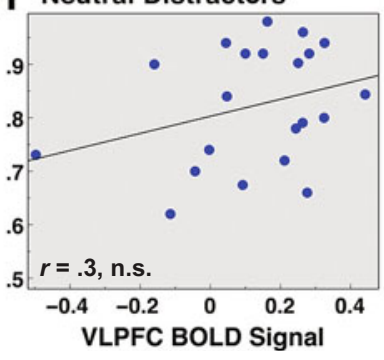

F Task-Related Distractors

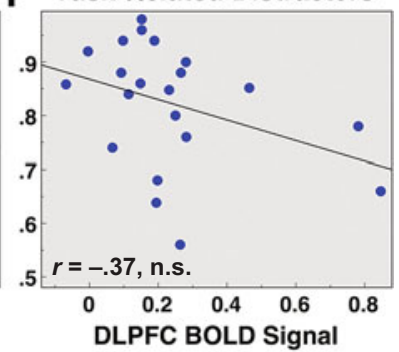

Figure 4. Individual differences in working memory (WM) performance as a function of prefrontal signal. Average WM performance (proportion correct) is shown as a function of average signal in PFC ROIs. (A-C) Frontopolar prefrontal cortex (aPFC) ROI is shown for negative $(r=-.52$, $p=.016$, two-tailed $)$, neutral $(r=-.04$, n.s. $)$, and task-related $(r=-.09$, n.s. $)$ distractor conditions; (D-F) DLPFC ROI is shown for negative $(r=-.67, p=.0004$, two-tailed), neutral $(r=-.29$, n.s.), and task-related $(r=-.37$, n.s.) distractor conditions; (G-I) VLPFC ROI is shown for negative $(r=$ -.09 , n.s.), neutral $(r=.3$, n.s.), and task-related $(r=-.23$, n.s.) distractor conditions. Results are collapsed across low and high WM load given the same pattern at both loads. PFC BOLD signal was averaged across both correct and incorrect trials.

As noted above, all three amygdala correlations were included along with cortical ROI correlations when controlling for FDR $(q=0.05)$ to ensure control of Type I error rate (Benjamini \& Hochberg, 1995). All three reported relationships for the amygdala exceeded their respective FDR thresholds.

\section{fcMRI Between the Amygdala and Other Cortical Regions During Resting State and Negative Distraction}

First, we examined the relationship between bilateral amygdala activity and activity in the rest of the brain in the absence of a task (resting state; Figure 7A). Figure 7A shows cortical regions that correlated significantly with the amygdala signal and met a whole-brain $p<.05$ correction
( $Z>3$ and 13 voxels, as determined by in-house Monte Carlo simulations). Overall, present results closely replicate prior work examining resting-state amygdala fcMRI (Roy et al., 2009), indicating significant negative coupling between amygdala and the main components of the dorsal task network (Corbetta et al., 2008). Second, we examined amygdala fcMRI in the context of WM function during negative distraction (Figure 7B), which indicated negative coupling with frontal, but not parietal, components of the dorsal task network, in line with fcMRI results reported by Mitchell et al. (2008). In addition, anterior cingulate and bilateral insula showed negative fcMRI with the amygdala during task, but not during resting state.

To examine which of these task and resting-state differences were statistically reliable, we computed an indepen- 

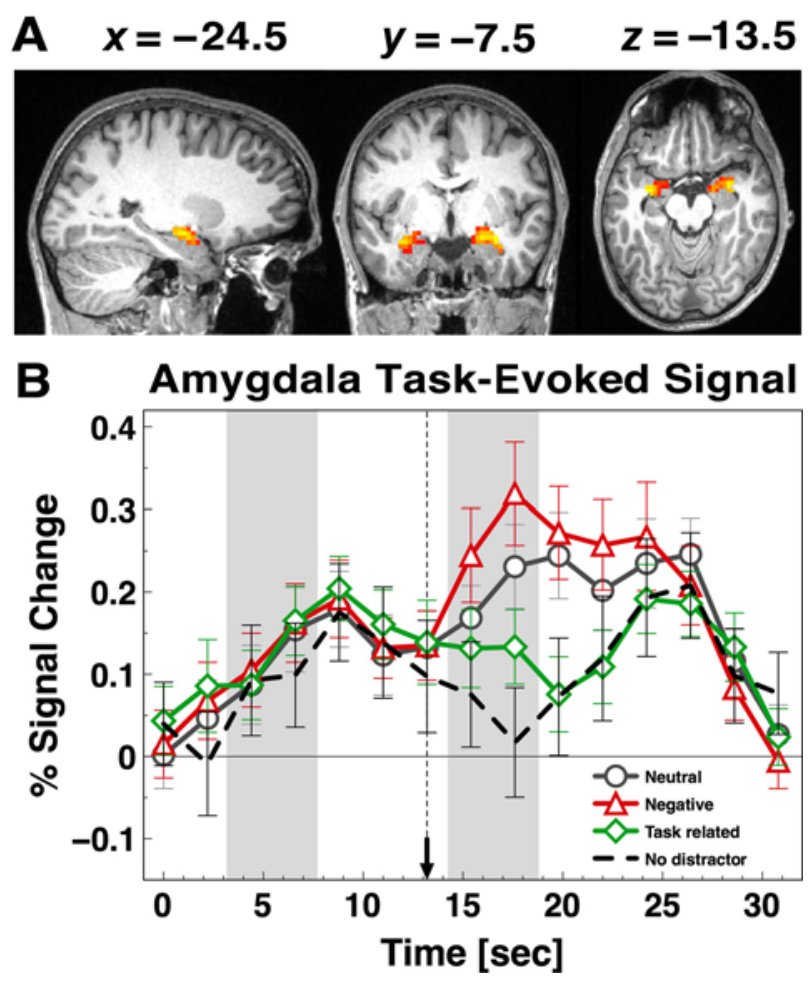

Figure 5. Bilateral amygdala signal. (A) Bilateral amygdala activation maps and (B) bilateral amygdala time courses are shown for neutral (circles), negative (triangles), task-related (diamonds), and no-distractor (dashed lines) conditions. Distractor onset is marked with a dotted vertical line ending in an arrow.

dent samples $t$ test using resting-state and task-based fcMRI results. The $t$ test results revealed significantly more negative coupling between the amygdala and prefrontal cortical regions during $\mathrm{WM}$ (with negative distraction) than during the resting state (Figure 7C, blue foci). These regions included bilateral DLPFC, bilateral aPFC, bilateral insular cortex, and bilateral anterior cingulate. In contrast, regions showing more positive coupling with the amygdala in task than in the resting state (Figure $7 \mathrm{C}$, red foci) seem to be largely centered around the posterior cortical regions, in- cluding the bilateral angular gyrus, and the bilateral sensory, visual, and posterior cingulate cortex. Of note, no prefrontal cortical foci showed more positive coupling with amygdala during task when compared with resting state. We also compared the amygdala fcMRI results from the negative distraction condition with those from the neutral distraction condition, using the same $t$ test approach. The whole-brain-corrected comparison between the two conditions (i.e., negative vs. neutral amygdala fcMRI) revealed differences centered on the same prefrontal regions showing more negative coupling in task versus rest (see Supplemental Figure S5F). Moreover, it is evident that at a somewhat lower threshold (i.e., $Z>2.5, p<.0065$ ), a wider set of regions very similar to those showing task-rest differences are also more negatively coupled with the amygdala during negative versus neutral distraction (see Supplemental Figures S5E and S5C).

Importantly, in the above analysis the subjects in the task-based fcMRI sample were different from those in the resting-state fcMRI sample. Thus, one concern is that the observed task versus rest results could have occurred for reasons having to do with sampling differences between groups of subjects, not ones reflecting differences in the specific factor of interest (in this case, task vs. resting state). In other words, maybe any two groups of randomly selected individuals would show differences in amygdala fcMRI under the exact same conditions (e.g., both resting state), instead of reflecting changes between task and resting-state fcMRI. To address this concern we employed a permutation resampling strategy (Nichols \& Holmes, 2001) that allowed us to determine whether the differences we observed were due to a specific way of examining the data (i.e., task vs. resting state) or whether some of these differences may have occurred due to chance alone, given a comparison of any two random sets of subjects (Hesterberg, Monaghan, Moore, Clipson, \& Epstein, 2005; see the supplemental materials for a complete discussion). Briefly, we computed 100,000 resampling simulations, which indicated that the differences observed in Figure 7C were unlikely to have occurred simply by randomly splitting the subjects into two groups. Supplemental Figure S5D shows the results of the permutation resampling.
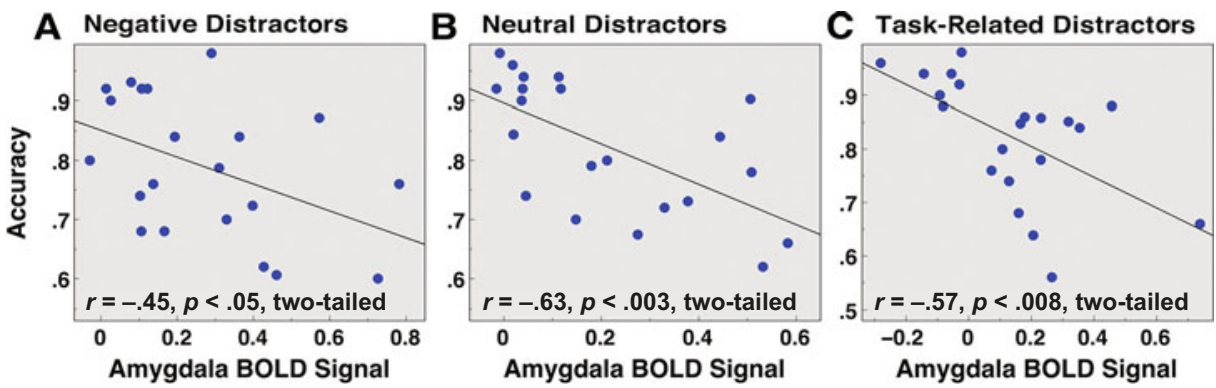

Figure 6. Individual differences in working memory (WM) performance as a function of amygdala signal. Average WM performance (proportion correct) is shown as a function of average bilateral amygdala signal for (A) negative $(r=-.45, p<.05$, two-tailed $)$; (B) neutral $(r=-.63, p<.003$, two-tailed); and (C) task-related $(r=-.57, p<.008$, two-tailed) distractor conditions. Results are collapsed across low and high WM load given the same pattern at both loads. Amygdala BOLD signal was averaged across both correct and incorrect trials. 
A Resting-State Amygdala fcMRI

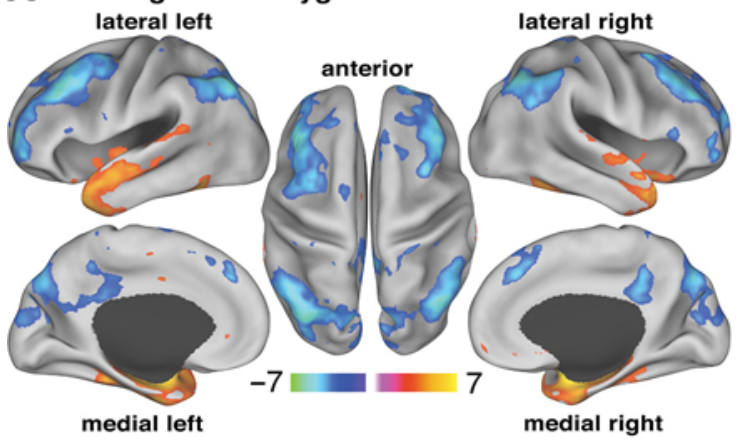

B Negative Distractor Task-Based Amygdala fcMRI

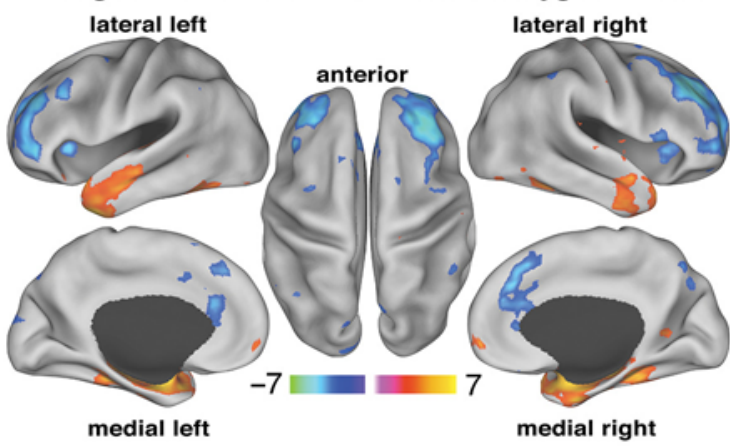

C Task-Based vs. Resting-State Amygdala fcMRI

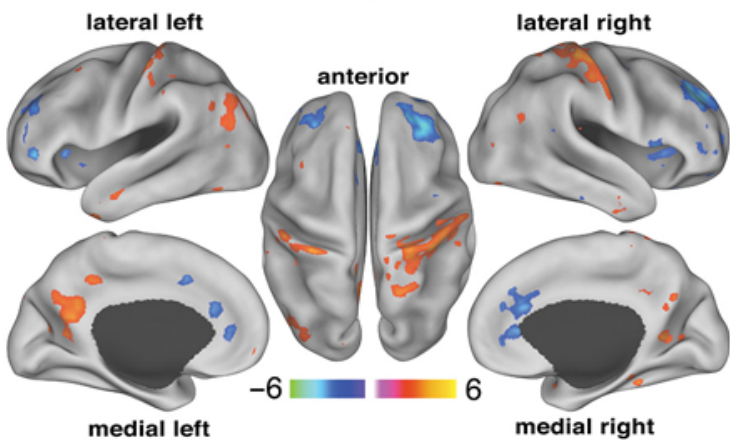

Figure 7. Amygdala resting-state and task-based functional connectivity. All maps are shown using $Z$ statistics and visualized using the PALS atlas (Van Essen, 2005). Bilateral amygdala fcMRI is shown (A) during resting state and (B) during WM faced with negative distraction. Brighter colors mark regions showing either more positive or more negative fcMRI with amygdala. The online version of this article shows positive and negative fcMRI with the amygdala in orange-yellow and blue colors, respectively. Both resting-state and task-based fcMRI maps show results corrected at whole-brain $p<.05$. (C) We also show results of an independent samples $t$ test comparing resting-state and task-based amygdala fcMRI. Here we show foci using a $Z>2.5$ threshold demonstrating that even with a lower cutoff more negative fcMRI for task versus resting state is centered mainly around the prefrontal nodes network and not elsewhere. The same foci are also shown using a whole-brain $p<.05$ correction in Supplemental Figure S5.

The voxels shown exceeded the observed task-rest difference in fewer than $0.1 \%(p<.001)$ of the simulations and closely correspond to the regions found using the independent samples $t$ test approach (also shown in Supplemental Figure S5C).

\section{DISCUSSION}

In the present study, we replicated prior work showing that negative distraction differentially modulates prefrontal activity during WM when compared with other types of distraction. Moreover, we extended prior findings in four important ways. We showed (1) that anterior prefrontal cortical regions modulated by emotion also evidenced performance-related activation differences specifically for negative distraction; (2) that a lower average signal in the dorsal PFC ROIs was associated with better WM performance across subjects, specifically during negative distraction; (3) that the amygdala was most responsive to negative distraction, but across subjects more amygdala signals during all distractor conditions were associated with poorer WM performance; and (4) that the amygdala was negatively coupled with frontal cortical regions during both resting state and active WM, but that this negative coupling with the prefrontal cortex was more prominent during negative distraction than during either resting state or neutral distraction.

\section{Negative Distraction Has an Impact on Prefrontal Cortical Regions}

The present study replicated and further validated previous findings in three prefrontal cortical regions, showing activity modulation as a function of emotion during WM (Dolcos \& McCarthy, 2006). In the absence of distraction, aPFC showed a sustained signal pattern during the maintenance phase marked by a prominent response to the probe, also reported by other groups (Leung et al., 2005). However, aPFC activation showed a below-baseline drop following negative distraction, and even more so when WM operations were not carried out successfully (i.e., incorrect trials), which was not apparent following neutral distraction. This aPFC region has been implicated in a variety of cognitive control functions, such as management of multiple task-relevant goals and sustained goal representation (Braver \& Bongiolatti, 2002; Braver, Reynolds, \& Donaldson, 2003; Dreher, Koechlin, Tierney, \& Grafman, 2008; Koechlin, Basso, Pietrini, Panzer, \& Grafman, 1999; Koechlin \& Hyafil, 2007; Reynolds, McDermott, \& Braver, 2006). One putative aPFC role put forth by Braver and Bongiolatti was that increased aPFC signals might reflect integration of subgoals during cognitive operations or "multitasking." Our results are consistent with this hypothesis, since aPFC exhibited the highest response to task-confusable distraction, which may require integration/comparison with the task. In other words, the similarity of the task-confusable distraction may require aPFC to carry out computations that aid in resolving interference arising from distractor-probe similarity (i.e., ignoring confusable distraction) while still allowing ongoing goal representation (i.e., maintaining memory set until the probe is presented). In line with this formulation, aPFC showed the greatest signal drop during negative distraction when WM was not carried out successfully (i.e., incorrect trials), which might suggest that trials in which a subgoal was processed (resolving emotion interference) led to loss or neglect of the primary task goal (memory set 
maintenance). Although speculative and in need of further testing, this interpretation is consistent with other models of aPFC function, suggesting that signal loss in this region may be associated with the loss of ongoing task goals (Koechlin \& Hyafil, 2007).

Similarly, DLPFC signals showed the greatest activity reduction when negative distraction was presented. However, the general pattern of DLPFC signals was different from that found in aPFC. In the absence of distraction, DLPFC showed a strong response during encoding (aPFC did not), above baseline signal during maintenance, then a clear response to the probe. This DLPFC region has typically been implicated in temporarily storing and manipulating information in the service of achieving a goal (Curtis et al., 2004; D'Esposito, 2007; Goldman-Rakic, 1996; Koechlin \& Hyafil, 2007). Thus, negative distraction may result in depleting available neural resources needed for adequate memory trace maintenance, resulting in a temporary signal drop. But, unlike aPFC, we did not observe that the amount of DLPFC signal drop was predictive of performance in the negative condition. One possibility is that in the present study the amount of negative distraction did not completely deplete DLPFC resources. Consistent with this interpretation, we did not observe below-baseline signal drop in DLPFC for negative distraction, as reported by Dolcos and McCarthy (2006), which may be due to differences in the amount of distraction presented (i.e., we used one distractor lasting for $1.1 \mathrm{sec}$, whereas they used two, lasting for $6 \mathrm{sec}$ ). Therefore, it may be possible that our emotional manipulation, although potent enough to produce an activation decrease and a behavioral effect, was not as capable of completely degrading memory traces held in DLPFC, and may be the reason we failed to observe a performance-related effect in DLPFC. It will be important for future studies to parametrically vary the amount of negative distraction to verify this assertion and establish at which level of negative distraction WM trace maintenance breaks down.

In contrast to aPFC and DLPFC, VLPFC showed a signal increase in response to negative but not to other salient distraction, also consistent with prior work (Dol$\cos \&$ McCarthy, 2006). Importantly, VLPFC signal increases were associated with better performance on trials containing negative, but not neutral, distraction, in line with prior studies (Dolcos et al., 2006). Prior work also showed that higher VLPFC signals were associated with lower distractibility ratings in the context of WM (Dolcos et al., 2006), as well as successful reappraisal of emotional information when no separate cognitive task was being performed (Ochsner \& Gross, 2005, 2008; Wager et al., 2008). Although other work has suggested that an elevated VLPFC signal may reflect a general role in interference control (Aaron, Robbins, \& Poldrack, 2004; ThompsonSchill et al., 2002), the present findings, and those of Dolcos et al. (2006), suggest that greater VLPFC activation may be uniquely associated with better WM performance when resolving negative distraction. Still, it is possible that negative distraction in our study produced more interference (or subjective sense of distraction) than did other distractors (as evident from the behavioral results), which may have in turn elicited the highest VLPFC activation. Although task-relevant distraction in the present study produced numerically more WM cost than did neutral distraction, it was not completely performance-matched with negative distractors. Therefore, to rule out this possibility, it will be critical for future studies to include a distractor condition devoid of emotion, but equally or more behaviorally distracting.

Lastly, we showed that lower aPFC and DLPFC signals, specifically during negative distraction, were associated with better WM performance across subjects. This was unexpected since - if anything - we would have expected that subjects with higher prefrontal recruitment during negative distraction would have performed better. One speculative explanation is that our findings reflect individual differences in WM capacity and its relationship with brain activation (Vogel \& Machizawa, 2004). In other words, low-capacity subjects may have reached, or were closer to, their neural recruitment plateau, whereas high-capacity subjects may have had resources to spare. Thus, higher capacity subjects may have shown relatively less PFC activation for the present task than did lower capacity subjects, but more resistance to distractions, due to better WM trace formation. Conversely, low-capacity subjects may have to rely on additional PFC recruitment to accomplish the task, but at the same time have fewer spare resources, possibly leading to stronger effects of negative distraction. Another speculative hypothesis is that, during negative distraction, certain subjects require far more PFC activity to overcome their elevated emotional reactivity and accomplish the WM task (e.g., higher trait anxiety; Bishop, 2009; Bishop, Duncan, Brett, \& Lawrence, 2004). These subjects may require increased aPFC and DLPFC recruitment to deal with the presence of negative interference, but more WM cost given stronger impact of affective material.

Alternatively, activation reductions in $\mathrm{PFC}$ regions may be linked to reallocating processing resources toward other brain regions involved in coping with emotional distraction (e.g., VLPFC). Therefore, higher reallocation may lead to more successful coping with emotional distraction, which in turn actually results in reductions of DLPFC activity. Future work using fcMRI analyses in this context may elucidate the nature of the relationship between DLPFC and other prefrontal regions, which are likely candidates in resisting emotional interference (e.g., VLPFC) and should do so when WM interference is resolved and successful reallocation may have taken place (i.e., correct vs. incorrect trials). In summary, because these findings were unexpected, further prospective testing is needed to investigate these competing hypotheses and characterize individual differences in PFC signals during affective distraction.

\section{Individual Differences in Amygdala Activation}

Consistent with our predictions, we demonstrated that, across subjects, higher amygdala amplitudes were associated with worse WM performance. However, this pattern was not specific for negative distraction, but was observed for all distractor types. Although the general relationship 
between WM performance and amygdala reactivity was somewhat surprising, it is not unprecedented. A study by Schaefer et al. (2006) demonstrated that higher amygdala amplitude was associated with faster RT during a challenging 3-back WM task devoid of emotion or distraction. Despite the evidence for the role of the amygdala as a central hub for detecting affective salience, numerous studies have implicated the amygdala in other nonaffective functions, such as attention and vigilance (Davis \& Whalen, 2001; Holland \& Gallagher, 1999, 2006; Kepp, Whalen, Supple, \& Pascoe, 1992; Sander, Grafman, \& Zalla, 2003). Accordingly, Schaefer and colleagues postulated that their findings might reflect the amygdala's role in general vigilance, which, in some contexts, may aid organisms in better coping with, and responding to, challenging cognitive conditions. Of note, Schaefer and colleagues found that increased amygdala response was associated with better, not worse, performance, as found in the present study. However, their findings reflected amygdala response to the probe in an $n$-back task, and not to distractors in the context of a delayed WM task. Amygdala responses, although facilitating vigilance during elevated cognitive challenge, may be detrimental at other times when increased vigilance may result in more potent distraction via external interference; in other words, whether higher amygdala responsiveness aids or interferes with task performance may differ, depending on the nature of the task involved.

Although the amygdala was maximally responsive to negative distraction, it also responded above baseline levels to other distractors (see Figure 5B). Therefore, given the present task, there may be a general expectation for a distractor occurring (emotional or not). In turn, certain subjects may have shown accompanied increases in amygdala recruitment, irrespective of distractor type. One speculative hypothesis is that there is some individual difference factor - trait anxiety, performance anxiety, distractibility - that leads some individuals to show stronger amygdala responses to any potentially performancerelevant distractor. It will be important for future work to elucidate which individual difference measures may predict increased amygdala amplitude, irrespective of distraction type, and to characterize contexts in which amygdala recruitment aids or hurts cognition.

Resting-state and task-based amygdala fcMRI. A main question was to investigate amygdala fcMRI during resting state and during WM faced with negative distraction. As noted by Roy et al. (2009), previous work has shown similar amygdala fcMRI during resting state and task (Stein et al., 2007), but has not directly tested whether there are fcMRI differences between task and rest or between the negative and the neutral task conditions. We replicate prior resting-state findings by showing negative fcMRI between the amygdala and the dorsal frontalparietal cortex, regions typically activated during effortful cognitive engagement (Corbetta et al., 2008; Curtis et al., 2004; D'Esposito et al., 1998; Dolcos \& McCarthy, 2006). In addition, we demonstrate that a number of regions located in the prefrontal cortex (but not elsewhere) showed more negative coupling with the amygdala during WM faced with negative distraction when compared with resting state, a subset of which also showed more negative coupling during negative versus neutral distraction. These regions included the bilateral DLPFC, the aPFC, the frontal operculum, and the dorsal anterior cingulate cortex, which - as noted - are in close correspondence with prefrontal components of the dorsal task network, as well as components of the cingulo-opercular system suggested by others as critical in maintaining stable set control (Dosenbach, Fair, Cohen, Schlaggar, \& Petersen, 2008; Dosenbach et al., 2007; Dosenbach et al., 2006). Interestingly, some of the same prefrontal circuits have been shown to come online during emotional reappraisal (Wager et al., 2008). One possibility is that certain prefrontal regions aid emotion regulation by suppressing amygdala signals during cognitive tasks such as WM. Importantly, a similar frontopolar region showing more negative coupling with the amygdala also showed the largest signal drop when WM operations failed in the face of negative distraction. Taken together, these converging findings point to the potential importance of the frontopolar cortex in resisting negative interference during cognitive engagement, possibly via down-regulating amygdala signals.

In addition, certain regions showed more positive fcMRI with the amygdala during negative distraction versus resting state; this included the visual cortex, the anterior temporal lobes, the angular gyrus, the posterior cingulate, and the somatosensory cortex. More positive coupling between the amygdala and these regions may reflect their increased interaction during processing of visually presented negative information. Other positive fcMRI changes, such as coupling with posterior cingulate and sensory cortex, were more surprising. Importantly, these differences did not reflect more positive coupling during task, but instead reflected less negative coupling in task versus resting state. These results were not predicted, and it is unclear at present what these specific changes may reflect. However, a critical point is that the majority of negative pictures depicted harm being inflicted (such as war footage, wounds, or mutilation pictures). Thus, one speculative hypothesis is that increased amygdala coupling with the somatosensory cortex may have reflected involvement of these regions in mental representation of pain infliction and internalizing the experience of the people in the pictures (Avenanti, Bueti, Galati, \& Aglioti, 2005; Avenanti, Minio-Paluello, Bufalari, \& Aglioti, 2006; Bufalari, Aprile, Avenanti, Di Russo, \& Aglioti, 2007; Cheng, Yang, Lin, Lee, \& Decety, 2008; Fecteau, Pascual-Leone, \& Théoret, 2008).

Of note, previously reported positive coupling between the amygdala and the VLPFC (Dolcos et al., 2006) was not replicated in the present study. One possibility is that the nature of the present task and the intensity of emotional distraction (i.e., Dolcos et al. [2006] used substantially more emotional distraction) required involvement of different mechanisms in coping with distraction, which may have diminished the role of VLPFC in suppressing amygdala signals. Lastly, we did not make predictions with regard to amygdala-prefrontal coupling as a function of accuracy. One possibility is that the degree 
of amygdala-prefrontal coupling changes depending on performance, which should be tested in prospective studies.

\section{Limitations and Future Directions}

Although we investigated the effect of negative emotional distraction on prefrontal activity during cognitive engagement, tests remain to be done to ascertain whether these effects are present during positively valenced distraction. Similarly, it would be informative to test whether other emotional material (i.e., verbal emotion or facial expressions) results in similar findings. It is also critical to point out that the present stimuli (negative vs. neutral) also differed along the arousal dimension, which may have contributed to the observed differences. While it is difficult to fully rule out, future work may want to use more carefully arousal-matched positive and negative distractors to verify the specificity of negative distraction found in the present study. Also, present fcMRI findings are correlational and do not address concerns related to directionality of influences. The use of converging methods that help establish causality (e.g., TMS), statistical techniques such as Granger causality (Bressler, Tang, Sylvester, Shulman, \& Corbetta, 2008), and pathway-mapping methods (Wager et al., 2008) will be integral to disambiguate the direction of specific regional influences during emotional distraction.

It should also be noted that the within-subjects accuracy analysis was based on a relatively small number of incorrect trials ( $\sim 10$ per condition). Therefore, it may be possible that a lack of significant differences in certain conditions and brain regions (e.g., neutral distraction for aPFC) was a product of low power and should be interpreted with this possibility in mind. Thus, future work should verify these effects using more difficult WM tasks with a larger number of error trials.

\section{CONCLUSION}

In the present study, we advanced the understanding of prefrontal cortex involvement in resisting negative distraction. We extended previous findings and showed that less frontopolar cortex deactivation, but more ventral lateral cortex activation, was associated with better WM performance, specifically during negative distraction. In addition, we showed that dorsal lateral and frontalpolar prefrontal regions demonstrated more negative coupling with the amygdala during negative distraction, when compared with resting state and neutral distraction. Lastly, we demonstrated that elevated amygdala signals were associated with worse WM performance, regardless of distraction type. Taken together, the present findings further establish the importance of prefrontal cortical circuitry in regulating temporary emotionally negative interference. Importantly, the present findings open important research venues for future investigations of clinical populations that may exhibit difficulties in either "top-down" (e.g., schizophrenia) or "bottom-up" (e.g., anxiety) circuitry critical for regulating negative emotions.

\section{AUTHOR NOTE}

This research was supported by NIMH Grant MH06603101 (D.M.B.), funded by the National Institute of Mental Health. We thank T. Braver for useful discussions, analyses, and suggestions. Please address correspondence to A. Anticevic, Department of Psychology, Campus Box 1125, Washington University, St. Louis, MO 63130 (e-mail: aanticev@, artsci.wustl.edu).

\section{REFERENCES}

Aaron, A. R., Robbins, T. W., \& Poldrack, R. A. (2004). Inhibition and the right inferior frontal cortex. Trends in Cognitive Sciences, $\mathbf{8}$, 170-177.

Attneave, F., \& Arnoult, M. D. (1956). The quantitative study of shape and pattern perception. Psychological Bulletin, 53, 452-471.

Avenanti, A., Bueti, D., Galati, G., \& Aglioti, S. M. (2005). Transcranial magnetic stimulation highlights the sensorimotor side of empathy for pain. Nature Neuroscience, 8, 955-960.

Avenanti, A., Minio-Paluello, I., Bufalari, I., \& Aglioti, S. M. (2006). Stimulus-driven modulation of motor-evoked potentials during observation of others' pain. NeuroImage, 32, 316-324.

Baddeley, A. D., \& Hitch, G. J. (1994). Developments in the concept of working memory. Neuropsychology, 8, 485-493.

Benjamini, Y., \& Hochberg, Y. (1995). Controlling the false discovery rate: A practical and powerful approach to multiple testing. Journal of the Royal Statistical Society B, 57, 125-133.

Bishop, S. J. (2009). Trait anxiety and impoverished prefrontal control of attention. Nature Neuroscience, 12, 92-98.

Bishop, S. [J.], Duncan, J., Brett, M., \& Lawrence, A. D. (2004). Prefrontal cortical function and anxiety: Controlling attention to threatrelated stimuli. Nature Neuroscience, 7, 184-188.

Bradley, M. M., Hamby, S., Löw, A., \& Lang, P. J. (2007). Brain potentials in perception: Picture complexity and emotional arousal. Psychophysiology, 44, 364-373.

Braver, T. S., \& Bongiolatti, S. R. (2002). The role of frontopolar cortex in subgoal processing during working memory. Neurolmage, 15, 523-536.

Braver, T. S., Reynolds, J. R., \& Donaldson, D. I. (2003). Neural mechanisms of transient and sustained cognitive control during task switching. Neuron, 39, 713-726.

Bressler, S. L., Tang, W., Sylvester, C. M., Shulman, G. L., \& CorBETTA, M. (2008). Top-down control of human visual cortex by frontal and parietal cortex in anticipatory visual spatial attention. Journal of Neuroscience, 28, 10056-10061.

Buckner, R. L., Head, D., Parker, J., Fotenos, A. F., Marcus, D., Morris, J. C., \& SNYDER, A. Z. (2004). A unified approach for morphometric and functional data analysis in young, old, and demented adults using automated atlas-based head size normalization: Reliability and validation against manual measurement of total intracranial volume. NeuroImage, 23, 724-738.

Bufalari, I., Aprile, T., Avenanti, A., Di Russo, F., \& Aglioti, S. M. (2007). Empathy for pain and touch in the human somatosensory cortex. Cerebral Cortex, 17, 2553-2561.

Cheng, Y., Yang, C. Y., Lin, C. P., Lee, P. L., \& Decety, J. (2008). The perception of pain in others suppresses somatosensory oscillations: A magnetoencephalography study. Neurolmage, 40, 1833-1840.

Collin, C. A., \& McMullen, P. A. (2002). Using Matlab to generate families of similar Attneave shapes. Behavior Research Methods, Instruments, \& Computers, 34, 55-68.

Corbetta, M., Patel, G., \& Shulman, G. L. (2008). The reorienting system of the human brain: From environment to theory of mind. Neuron, 58, 306-324.

Corbetta, M., \& Shulman, G. L. (2002). Control of goal-directed and stimulus-driven attention in the brain. Nature Reviews Neuroscience, 3, 201-215.

Curtis, C. E., Rao, V. Y., \& D’Esposito, M. (2004). Maintenance of spatial and motor codes during oculomotor delayed response tasks. Journal of Neuroscience, 24, 3944-3952.

Davis, M., \& Whalen, P. J. (2001). The amygdala: vigilance and emotion. Molecular Psychiatry, 6, 13-34.

Delplanque, S., N'diaye, K., Scherer, K., \& Grandjean, D. (2007). Spatial frequencies or emotional effects? A systematic measure of 
spatial frequencies for IAPS pictures by a discrete wavelet analysis. Journal of Neuroscience Methods, 165, 144-150.

Desimone, R., \& Duncan, J. (1995). Neural mechanisms of selective visual attention. Annual Review of Neuroscience, 18, 193-222.

D'Esposito, M. (2007). From cognitive to neural models of working memory. Philosophical Transactions of the Royal Society B, 362, 761772 .

D’Esposito, M., Aguirre, G. K., Zarahn, E., Ballard, D., Shin, R. K., \& LEASE, J. (1998). Functional MRI studies of spatial and nonspatial working memory. Cognitive Brain Research, 7, 1-13.

Dolcos, F., Diaz-Granados, P., Wang, L., \& McCarthy, G. (2008). Opposing influences of emotional and non-emotional distracters upon sustained prefrontal cortex activity during a delayed-response working memory task. Neuropsychologia, 46, 326-335.

Dolcos, F., Kragel, P., Wang, L., \& McCarthy, G. (2006). Role of the inferior frontal cortex in coping with distracting emotions. NeuroReport, 17, 1591-1594.

Dolcos, F., \& McCarthy, G. (2006). Brain systems mediating cognitive interference by emotional distraction. Journal of Neuroscience, 26, 2072-2079.

Dolcos, F., Miller, B., Kragel, P., Jha, A., \& McCarthy, G. (2007). Regional brain differences in the effect of distraction during the delay interval of a working memory task. Brain Research, 1152, 171-181.

Dosenbach, N. U. F., Fair, D. A., Cohen, A. L., Schlaggar, B. L., \& Petersen, S. E. (2008). A dual-networks architecture of top-down control. Trends in Cognitive Sciences, 12, 99-105.

Dosenbach, N. U. F., Fair, D. A., Miezin, F. M., Cohen, A. L., Wenger, K. K., Dosenbach, R. A. T., ET AL. (2007). Distinct brain networks for adaptive and stable task control in humans. Proceedings of the National Academy of Sciences, 104, 11073-11078.

Dosenbach, N. U. F., Visscher, K. M., Palmer, E. D., Miezin, F. M., Wenger, K. K., KANG, H. C., ET AL. (2006). A core system for the implementation of task sets. Neuron, 50, 799-812.

Dreher, J. C., Koechlin, E., Tierney, M., \& Grafman, J. (2008). Damage to the fronto-polar cortex is associated with impaired multitasking. PLoS One, $\mathbf{3}$, e3227.

Fecteau, S., Pascual-Leone, A., \& Théoret, H. (2008). Psychopathy and the mirror neuron system: Preliminary findings from a nonpsychiatric sample. Psychiatry Research, 160, 137-144.

Fischl, B., Salat, D. H., Busa, E., Albert, M., Dieterich, M., HaselGROVE, C., ET AL. (2002). Whole brain segmentation: Automated labeling of neuroanatomical structures in the human brain. Neuron, $\mathbf{3 3}$, 341-355.

Fischl, B., Salat, D. H., van der Kouwe, A. J., Makris, N., SéGONNE, F., QuinN, B. T., ET AL. (2004). Sequence-independent segmentation of magnetic resonance images. NeuroImage, 23, S69-S84.

Fox, M. D., Snyder, A. Z., Vincent, J. L., Corbetta, M., VAn EsSEn, D. C., \& RaIchle, M. E. (2005). The human brain is intrinsically organized into dynamic, anticorrelated functional networks. Proceedings of the National Academy of Sciences, 102, 9673-9678.

GoldMAN-RAKIC, P. S. (1996). The prefrontal landscape: Implications of functional architecture for understanding human mentation and the central executive. In A. C. Roberts, T. W. Robbins, \& L. Weiskrantz (Eds.), The prefrontal cortex: Executive and cognitive functions (pp. 87-103). Oxford: Oxford University Press.

He, B. J., Snyder, A. Z., Vincent, J. L., Epstein, A., Shulman, G. L., \& Corbetta, M. (2007). Breakdown of functional connectivity in frontoparietal networks underlies behavioral deficits in spatial neglect. Neuron, 53, 905-918.

Hesterberg, T., Monaghan, S., Moore, D. S., Clipson, A., \& EpSTEIN, R. (2005). Bootstrap methods and permutation tests (2nd ed.). New York: Freeman.

Holland, P. C., \& Gallagher, M. (1999). Amygdala circuitry in attentional and representational processes. Trends in Cognitive Sciences, 3, 65-73.

Holland, P. C., \& Gallagher, M. (2006). Different roles for amygdala central nucleus and substantia innominata in the surprise-induced enhancement of learning. Journal of Neuroscience, 26, 3791-3797.

Kepp, B. S., Whalen, P. J., Supple, W., \& Pascoe, J. P. (1992). Amygdaloid contributions to conditioned arousal and sensory information processing. In J. P. Aggleton (Ed.), The amygdala: Neurobiological aspects of emotion, memory, and mental dysfunction (pp. 229-254). New York: Wiley.
Kerr, D. L., Gusnard, D. A., Snyder, A. Z., \& Raichle, M. E. (2004). Effect of practice on reading performance and brain function. NeuroReport, 15, 607-610.

Koechlin, E., Basso, G., Pietrini, P., Panzer, S., \& Grafman, J. (1999). The role of the anterior prefrontal cortex in human cognition. Nature, 399, 148-151.

Koechlin, E., \& Hyafil, A. (2007). Anterior prefrontal function and the limits of human decision-making. Science, 318, 594-598.

LANG, P. J., Bradley, M. M., \& Cuthbert, B. N. (1999). International affective picture system (IAPS): Affective ratings of pictures and instruction manual. Gainesville: University of Florida.

LANG, P. J., \& Davis, M. (2006). Emotion, motivation, and the brain: Reflex foundations in animal and human research. Progress in Brain Research, 156, 3-29.

LeDoux, J. E. (2000). Emotion circuits in the brain. Annual Review of Neuroscience, 23, 155-184.

Leung, H. C., Gore, J. C., \& Goldman-Rakic, P. S. (2005). Differential anterior prefrontal activation during the recognition stage of a spatial working memory task. Cerebral Cortex, 15, 1742-1749.

Michelon, P., Snyder, A. Z., Buckner, R. L., McAvoy, M., \& Zacks, J. M. (2003). Neural correlates of incongruous visual information: An event-related fMRI study. NeuroImage, 19, 1612-1626.

Miller, B. T., \& D'Esposito, M. (2005). Searching for "the top" in top-down control. Neuron, 48, 535-538.

Miller, E. K., \& CoHEN, J. D. (2001). An integrative theory of prefrontal cortex function. Annual Review of Neuroscience, 21, 167-202.

Mitchell, D. G., Luo, Q., Mondillo, K., Vythilingam, M., Finger, E. C., \& BLAIR, R. J. (2008). The interference of operant task performance by emotional distracters: An antagonistic relationship between the amygdala and frontoparietal cortices. NeuroImage, 40, 859-868.

Morris, J. S., Öhman, A., \& Dolan, R. J. (1998). Conscious and unconscious emotional learning in the human amygdala. Nature, 393, 467-470.

Morris, J. S., Öhman, A., \& Dolan, R. J. (1999). A subcortical pathway to the right amygdala mediating "unseen" fear. Proceedings of the National Academy of Sciences, 96, 1680-1685.

Nichols, T. E., \& Holmes, A. P. (2001). Nonparametric permutation tests for functional neuroimaging: A primer with examples. Human Brain Mapping, 15, 1-25.

Ochsner, K. N., Bunge, S. A., Gross, J. J., \& Gabrieli, J. D. E. (2002). Rethinking feelings: An fMRI study of the cognitive regulation of emotion. Journal of Cognitive Neuroscience, 14, 1215-1229.

OCHSNER, K. N., \& Gross, J. J. (2005). The cognitive control of emotion. Trends in Cognitive Sciences, 9, 242-249.

OChSNER, K. N., \& Gross, J. J. (2008). Cognitive emotion regulation: Insights from social cognitive and affective neuroscience. Current Directions in Psychological Science, 17, 153-158.

Ochsner, K. N., Hughes, B., Robertson, E. R., Cooper, J. C., \& GABRIELI, J. D. E. (2009). Neural systems supporting the control of affective and cognitive conflicts. Journal of Cognitive Neuroscience, 21, 1841-1854

Öhman, A. (2005). The role of the amygdala in human fear: Automatic detection of threat. Psychoneuroendocrinology, 30, 953-958.

Öhman, A., Flykt, A., \& Esteves, F. (2001). Emotion drives attention: Detecting the snake in the grass. Journal of Experimental Psychology: General, 130, 466-478.

Ojemann, J., Akbudak, E., Snyder, A., McKinstry, R., Raichle, M., \& Conturo, T. (1997). Anatomic localization and quantitative analysis of gradient refocused echo-planar fMRI susceptibility artifacts. NeuroImage, 6, 156-167.

Ollinger, J. M., Corbetta, M., \& Shulman, G. L. (2001). Separating processes within a trial in event-related functional MRI. NeuroImage, 13, 218-229.

PESSOA, L. (2005). To what extent are emotional visual stimuli processed without attention and awareness? Current Opinion in Neurobiology, 15, 188-196.

PessoA, L. (2008). On the relationship between emotion and cognition. Nature Reviews Neuroscience, 9, 148-158.

Pessoa, L., JAPEe, S., \& UngerLeider, L. G. (2005). Visual awareness and the detection of fearful faces. Emotion, 5, 243-247.

Phan, K. L., Wager, T. D., TAYlor, S. F., \& Liberzon, I. (2004). Functional neuroimaging studies of human emotions. CNS Spectrums, $\mathbf{9}$, 258-266. 
Phelps, E. A. (2006). Emotion and cognition: Insights from studies of the human amygdala. Annual Review of Psychology, 57, 27-53.

Phelps, E. A., \& LeDoux, J. E. (2005). Contributions of the amygdala to emotion processing: From animal models to human behavior. Neuron, 48, 175-187.

Reynolds, J. R., McDermott, K. B., \& Braver, T. S. (2006). A direct comparison of anterior prefrontal cortex involvement in episodic retrieval and integration. Cerebral Cortex, 16, 519-528.

Roy, A. K., Shehzad, Z., Margulies, D. S., Kelly, A. M., Uddin, L. Q., Gotimer, K., ET AL. (2009). Functional connectivity of the human amygdala using resting state fMRI. NeuroImage, 45, 614-626.

Sabatinelli, D., Bradley, M. M., Fitzsimmons, J. R., \& Lang, P. J. (2005). Parallel amygdala and inferotemporal activation reflect emotional intensity and fear relevance. NeuroImage, 24, 1265-1270.

Sander, D., Grafman, J., \& Zalla, T. (2003). The human amygdala: An evolved system for relevance detection. Reviews in the Neurosciences, 14, 303-316.

Schaefer, A., Braver, T. S., Reynolds, J. R., Burgess, G. C., YARKONI, T., \& GRAY, J. R. (2006). Individual differences in amygdala activity predict response speed during working memory. Journal of Neuroscience, 26, 10120-10128.

Stein, J. L., Wiedholz, L. M., Bassett, D. S., Weinberger, D. R., Zink, C. F., Mattay, V. S., \& Meyer-Lindenberg, A. (2007). A validated network of effective amygdala connectivity. NeuroImage, 36, 736-745.

STERnBerG, S. (1969). The discovery of processing stages: Extensions of Donders' method. In W. G. Koster (Ed.), Attention and performance II (pp. 276-315). Amsterdam: North-Holland.

TAlairach, J., \& Tournoux, P. (1988). Co-planar stereotaxic atlas of the human brain: 3-dimensional proportional system: An approach to cerebral imaging (M. Rayport, Trans.). New York: Thieme.

Thompson-Schill, S. L., Jonides, J., Marshuetz, C., Smith, E. E.,
D'Esposito, M., Kan, I. P., ET AL. (2002). Effects of frontal lobe damage on interference effects in working memory. Cognitive, Affective, \& Behavioral Neuroscience, 2, 109-120.

VAN EsSEN, D. C. (2005). A population-average, landmark- and surfacebased (PALS) atlas of human cerebral cortex. NeuroImage, 28, 635662.

Vogel, E. K., \& Machizawa, M. G. (2004). Neural activity predicts individual differences in visual working memory capacity. Nature, 428, 748-751.

Vuilleumier, P. (2005). How brains beware: Neural mechanisms of emotional attention. Trends in Cognitive Sciences, 9, 585-594.

Vuilleumier, P., \& Pourtois, G. (2007). Distributed and interactive brain mechanisms during emotion face perception: Evidence from functional neuroimaging. Neuropsychologia, 45, 174-194.

Wager, T. D., Davidson, M. L., Hughes, B. L., Lindquist, M. A., \& OCHSNER, K. N. (2008). Prefrontal-subcortical pathways mediating successful emotion regulation. Neuron, 59, 1037-1050.

Wager, T. D., Phan, K. L., Liberzon, I., \& TaYlor, S. F. (2003). Valence, gender, and lateralization of functional brain anatomy in emotion: A meta-analysis of findings from neuroimaging. Neurolmage, 19, 513-531.

ZALD, D. H. (2003). The human amygdala and the emotional evaluation of sensory stimuli. Brain Research Reviews, 41, 88-123.

\section{SUPPLEMENTAL MATERIALS}

The supplemental figures and table referred to in this article, as well as additional information about the methods, may be downloaded from http://cabn.psychonomic-journals.org/content/supplemental.

(Manuscript received June 18, 2009;

revision accepted for publication September 21, 2009.) 